\title{
Max Brod als Prager Kulturmittler
}

\author{
Konzepte des Kulturtransfers um 1918 \\ zwischen Profession und Mission
}

\section{STEFFEN HÖHNE}

\begin{abstract}
Today Max Brod is primarily known because of his friend Franz Kafka, whose works, for a large part, he was able to preserve for posterity. As a consequence, his own extensive literary texts have almost completely fallen into oblivion. But also his intermediation activities, which included Czech, German and Jewish artists and their art, were of immense cultural and political importance. This paper will point to the opportunities but also limitations of Max Brod's transnational and transcultural intermediation activities.
\end{abstract}

Title: Max Brod as a Cultural Intermediator in Prague. Concepts of Cultural Transfer around 1918 between Profession and Mission

Keywords: cultural transfer; Prague German literature; Brod, Max (1884-1968); cultural policy; antisemitism

\section{VORBEMERKUNGEN}

Max Brod dürfte vielen, anders als sein Freund Franz Kafka, weitgehend unbekannt sein, zumal seine literarischen und essayistischen Werke auf dem Buchmarkt lange vergriffen waren. ${ }^{1}$ Dabei war Max Brod eine der zentralen Gestalten in Prag bzw. Mitteleuropa, die Engagement und Verantwortung sowohl in politischer wie in kultureller und sprachlicher Hinsicht als Maxime ihres intellektuellen Handelns ansahen und die gewissermaßen einen neuen Typus des heimatlosen Weltbürgers - oft jüdischer Abstammung - verkörperten. Speziell für die mitteleuropäischen und somit auch Prager Intellektuellen nach 1900 scheinen Reflexionen kulturell-sprachlicher Identifikation und kulturellen Transfers eine zentrale Komponente im Denken darzustellen. Intellektuelle und Künstler wie Max Brod sind Begründer einer neobohemistischen Tradition, die in der Kafka-Zeit einzusetzen begann. ${ }^{2}$ Parallel zu früh-aktivistischen,

1 Derzeit wird eine zehnbändige Werkausgabe Max Brods im Wallstein-Verlag vorbereitet, hg. v. Hans-Gerd Koch u. Hans-Dieter Zimmermann.

2 | Unter Bohemismus wird ein Integrationsmodell für die böhmischen Länder vor 1848 verstanden, welches die nationalen Divergenzen und Interessen zwischen Tschechen und Deutschen zugunsten übernationaler Einstellungen aufzulösen suchte und dabei von einer prinzipiellen Gleichheit im Sinne einer nicht-prioritären, auch sprach- 
meist in austriazistischen Traditionskontexten eingebetteten Konzeptionen, die sich z.B. bei Franz Spina vor 1918 finden lassen (vgl. Höhne 2012), zeigen sich im kulturell-literarischen Diskurs in dieser Zeit ebenfalls neobohemistische Positionierungen, die zunächst in literarisch-künstlerischen Kreisen einsetzten und - bezogen auf Prag - in der Zeit zwischen 1911 und dem Ersten Weltkrieg einen Höhepunkt erlebten (vgl. Krolop 2005), wobei es schon zuvor im Kontext der sich herausbildenden Moderne in den böhmischen Ländern etwa ab Mitte der 18 goer-Jahre zu einer zumindest partiellen kulturellen Annäherung kam (vgl. Schmitz/Udolph 2001). Publizistisch durch deutschsprachige Periodika wie die Herder-Blätter (1911/12) flankiert, trat eine kleine Gruppe von Intellektuellen und Künstlern an, um die bestehende Polarisierung zwischen Tschechen und Deutschen in Prag zu durchbrechen und auf einen Ausgleich zwischen den nationalen Gruppen hinzuwirken. ${ }^{3}$ Damit war auch eine Neupositionierung jener Gruppe intendiert, die später als Prager Kreis weltberühmt werden sollte, für die Franz Werfel am 18. April 1914 im Prager Tagblatt in seiner »Glosse zu einer Wedekind-Feier« programmatisch formulierte: »Wir aber wollen wieder teilnehmen an Prag.«(Zit. n. Krolop 2005: 68f.)

Insbesondere unter den Prager jüdischen Künstlern, meist Autoren, entwickelten sich in der Folge intensive Debatten um kulturelle Identität, Alterität und Vermittlung in der Dreivölkerstadt Prag (vgl. Tramer 1964), die zwischen assimilatorischen deutsch- oder tschechojüdischen sowie zionistischen Optionen verliefen, gerahmt von austropetalen Orientierungen (vgl. Werfel 1980: 320) einer postnationalen, transkulturellen oder auch utraquistischen Moderne bzw. dem Modell der jüdischen Diaspora, wie es Brod 1918 noch vor der Gründung der Tschechoslowakei auch im Hinblick auf seine soziale Position formulierte:

Ich fühle mich nicht als Angehöriger des deutschen Volkes, doch bin ich ein Freund des Deutschtums und außerdem durch Sprache und Erziehung, durch vieles von dem, was die Soziologie [...] 'Traditionswerte، im Gegensatz zu ,Generationswerten` (Erbwerten) nennt, dem Deutschtum kulturverwandt. Ich bin ein Freund des Tschechentums und im Wesentlichen [...] dem Tschechentum kulturfremd. (Brod 1918: 1581)

lichen Gleichberechtigung der Böhmen sslawischen wie deutschen Stammesı ausging. Eine spätbohemistische Phase schließt sich bis 1866/67 an, ihren Höhepunkt und ihr Ende markiert Adalbert Stifters Roman Witiko. Mit dem Einsatz der Prager Moderne (1894/95) verlaufen Ansätze neobohemistischer Einstellung, die nach 1918 teilweise mit dem politischen Aktivismus korrelieren (vgl. Höhne 2000).

3 Siehe hierzu die Beiträge Krolops 2005, ferner Godé/Le Rider/Mayer 1996, Höhne/Udolph 2010 sowie Becher/Höhne/Nekula 2012. 


\section{Spezifika des Kulturtransfers in Prag}

Ausgehend von einer Rahmenanalyse in der Tradition Erving Goffmans (1993), wonach spezifische sozialhistorische, kulturelle, mentale und ideologische Rahmen die Wahrnehmung prägen, lässt sich die wachsende nationale Polarisierung und die damit verbundene Entfremdung zwischen den gebildeten Schichten, für die Konzepte exkludierender Integration, sinnfällig im Leitmotto »Svůj k svému« (»Jeder zu seinem Eigenen«), zum Maßstab politischer, kultureller und sprachlicher Orientierung wurden, als ein solcher Rahmen betrachten, auf den die Kulturmittler reagierten bzw. reagieren mussten. Was für eine Gruppe waren nun diese Kulturmittler, sofern man überhaupt von einer Gruppe sprechen kann? Die Benennung Kultur und Vermittlung verweist auf Ansätze der Kulturtransferforschung bzw. auf Konzepte aus der interkulturellen Kommunikationsforschung (vgl. Werner/Zimmermann 2002). Ausgehend von den Objekten, Praktiken, Texten und Diskursen einer Ausgangskultur initiieren die Kulturmittler als Vermittlungsinstanzen einen im Idealfall wechselseitigen Transfer mit der jeweils anderen Kultur als Zielkultur, wobei bestimmte Selektionsmodi (was wird vermittelt?) sowie Formen der Aneignung und produktiven Rezeption zu einer Annäherung zwischen zwei oder mehr Kulturen beitragen sollen (vgl. Koeltzsch 2011). Vermittlerfiguren und -instanzen sind dabei formal betrachtet vor allem Autoren, Übersetzer, Verleger, z. T. die Medien, theoretisch auch Bildungsinstitutionen wie Schulen und Universitäten oder Institutionen aus dem kulturellen Feld, die allerdings in dem hier zu betrachtenden Zeitraum, der Kafka-Zeit, oftmals der Logik einer eigennational konnotierten Sammlung und Integration bei strikter Abgrenzung vom anderskulturellen Gegner verpflichtet zu sein schienen (vgl. Němec 2010). Findet man auch für den fraglichen Zeitraum typische Textsorten, neben Anthologien vor allem Periodika, Enzyklopädien, Übersetzungen, Lesebücher, bildungs-, kultur-, sprach-, wissenschaftspolitische Essays bis hin zu Reiseführern und sonstigen Ratgebern, in denen es zu einer expliziten Thematisierung des Zusammenlebens von Deutschen, Tschechen und/oder Juden in den böhmischen Ländern oder im weiteren mitteleuropäischen Kontext kommt, so soll damit keine homogene Gruppe von Kulturmittlern oder auch nur im Dienste der Vermittlung stehenden Texten konstruiert werden. Gleichwohl zeigt sich in der Summe dieser kulturvermittelnden Aktivitäten ein übergreifender Vermittlungsanspruch bzw. Habitus, der allerdings angesichts dominanter ideologisch-nationalistischer Diskurse ein - wenngleich wirkungsmächtiges - Randphänomen innerhalb der übermächtigen nationalen Narrative darstellt, weshalb die Kulturmittler in der Regel aus dem nationalen Kanon ausgegrenzt wurden. Insofern hängt, dies sei am Rande vermerkt, ihre nicht nur wissenschaftliche Wiederentdeckung häufig erst mit der Etablierung postkolonialer Studien sowie der Kulturtransferforschung zusammen, mit denen sich allmählich die Erkenntnis durchzusetzen beginnt, dass Kulturen nicht als einheitliche und klar abgrenzbare Gebilde so der essentialistische Zugang - zu verstehen sind, sondern als Ensembles kontextabhängiger und immer situativ bedingter Verhaltensweisen, Wahrneh- 
mungs- und Deutungsmuster. Kulturen sind immer schon vermischt, prozessual organisiert, wandelbar und verfügen über keine aus irgendeiner Entität zu begründende Valenz.

Im Gegensatz hierzu werden ethnische Kategorien und Zuordnungen wie $>$ Rasse < oder Ethnie von den Kulturmittlern, so auch von dem hier im Zentrum stehenden Max Brod, in keiner Weise verworfen, sondern bestenfalls nivelliert, denn dass

Abkömmlinge aus deutschen Häusern wie Rieger, Fügner usw. prominente Tschechen geworden sind, spricht [...] natürlich nur für die rassenhafte Verwandtschaft oder vielleicht beiderseitige Rassenunbestimmtheit der Deutschen und Tschechen, beweist aber für das rassenmäßig so fest umschriebene, seit Jahrtausenden unvermischte Judentum nicht das Geringste. (Brod 1918: 1589)4

\section{Brod als Kulturmittler - Voraussetzungen}

Max Brod, den die germanistische Forschung vor allem als engsten Freund und Nachlassverwalter Kafkas und Herausgeber von dessen Werken tradiert, war zweifellos der erfolgreichste Kulturmittler im deutsch-tschechisch-jüdischen Kontext des 20. Jahrhunderts. Sein Nachruhm basiert weniger auf seinen eigenen literarischen Werken als auf seiner Vermittlungstätigkeit, die im Hinblick auf Kafka, Janáček, Hašek - und dies ungeachtet späterer Kritik an seiner Editions- und Übersetzungstätigkeit ${ }^{5}$ - von nicht zu unterschätzender Bedeutung sein dürfte.

Brod ging es bei seinen Vermittlungsaktivitäten zunächst um den Aufbau von Netzwerken und zentrierenden Periodika (Prager Kreis, Arkadia 1913), um Positionierung und Abgrenzung (z.B. die Polemik gegen Karl Kraus 1911), wobei sich im Umfeld des Ersten Weltkriegs eine Verlagerung abzeichnet vom Kreis der Prager deutschen Autoren, die zu einem großen Teil Prag verlassen, ${ }^{6}$ zur tschechischen Kultur. Brod setzte sich dabei intensiv mit der tschechischen Kultur auseinander. Seit 1907 stellte er in deutschsprachigen Medien regelmäßig tschechische Künstler vor, seit 1909 erschienen Artikel zur tschechischen

4 Insofern lassen sich die neobohemistischen Einstellungsmuster nur bedingt mit dem supranationalen und landespatriotischen des Bohemismus vor 1848 in Einklang bringen. Jindřich (ursprünglich Heinrich Anton) Fügner (1822-1865) war Mitbegründer des tschechischen Turnvereins Sokol; František Ladislav (Franz Ladislaus) Rieger (1818-1903) war führender Politiker der Alltschechischen Partei. Brod spielt hier auf den Wechsel von der deutschen in die tschechische Kultur und Sprache an.

5 | Hierzu ausführlich Šrámková 2010: 293-305.

6 | Dieser "Differenzierungs- und Abwanderungsprozess, der um die Jahrhundertwende begonnen hatte" (Krolop 2005: 89), wird in einer Umfrage Warum haben Sie Prag verlassen? sinnfällig, die in der Deutschen Zeitung Bohemia und dem Prager Tagblatt Ende Mai und Anfang Juni 1922 veröffentlicht wurde. 
Musik (Dvořák und Smetana), mit denen der Blick des deutschen Publikums auch auf unbekannte Komponisten wie Josef $\operatorname{Suk}^{7}$ und Vitězlav Novák ${ }^{8}$ gelenkt werden sollte (vgl. Vassogne 2009: 195f.).

Brod, der sich bis in den Ersten Weltkrieg hinein als Austriazist bekannte (Brod 1918 u. 1979: 98f.) - noch 1918 beschwört er »eine gerechte Föderationsregierung autonomer Völker« (Brod 1918: 1584f.) sowie eine wirkliche »Völkerautonomie« als Ausgleich »deutscher, tschechischer und jüdischer Politik« entwickelte in der Folge als ideelle Grundlage seiner auch kulturpolitischen Vermittlertätigkeit Modelle wie den Nationalhumanismus und die Distanzliebe. Mit dem Konzept des demokratisch legitimierten Nationalhumanismus skizzierte Brod eine Art gereinigten Nationalismus:

Allerdings ist mein Nationalbegriff ein gründlich anderer als der heute selbst bei sogenannten gemäßigten Nationalisten herrschende National-Imperialismus. Mein Nationalismus faßt die Möglichkeit, ja die Pflicht freiwilligen Opfers nationaler Interessen zugunsten der Menschheit in sich [...]. Mit aller Schärfe muß diesem heutigen Nationalismus, der den Kultur- und Geisteswert des Volkstums zu kapitalistischer Pleonexie vergewaltigt, ein neuer Nationalismus entgegengesetzt werden. (Ebd.: 1591f.)

Ausgehend von der Situation der kleinen Völker, deren Verhältnisse auf einer Friedenskonferenz neu zu regeln wären, bei der auch das jüdische Volk seine Stimme erheben werde, skizziert Brod eine neue Interessenallianz zwischen Zionismus und tschechischer Emanzipation:

Ein politisches Zusammenarbeiten der Juden und Tschechen ist möglich; namentlich in Österreich, wo auch wir Juden die weitgehendste nationale Autonomie der kleinen Nationen anstreben. In dieser Beziehung sind die Deutschen unsere Feinde. (Brief v. 30. Juli 1917, zit. n. Racek/Rektorys 1935: 35) ${ }^{9}$

Das Utopische dieses Nationalhumanismus bald erkennend - man denke nur an die politisch-chauvinistische, antisemitische Radikalisierung auch auf tschechischer Seite nach 1918, ${ }^{10}$ vor allem in Gestalt der >Prager Ereignisse< vom 16. bis 19. November 1920, denen man wiederum einen der seltenen unmittelbar politischen Reflexe Kafkas verdankt (Brief an Milena, Mitte November 1920; Kafka 2013: 390) - gelangt Brod zu einer neuen, zionistischen Positionierung,"

7 | Zu Suk s. Šrámková 2010: 51-60.

8 | Zu Novák s. Šrámková 2010: 57-62.

9 | Diese Position wird auch demografisch und somit machtpolitisch begründet: "Wird der Zionismus nicht gerade aus diesem Grund von den Tschechen freundlich beurteilt, weil er durch Bekenntnis zur jüdischen Nationalität die Zahl der Deutschen in Prag und andern tschechischen Orten mit deutschsprechender Minorität verringert?" (Brod 1918: 1583)

10 | Zum tschechischen Antisemitismus um 1900 s. zuletzt Frankl 2011.

11 | Die Identitätssuche Brods verlief zunächst über die Schopenhauer-Rezeption, an die sich Brods Indifferentismus anschloss, bis er kurz vor dem Ersten Weltkrieg das 
mit der die eigene Gruppe von Deutschen und Tschechen gleichermaßen abgegrenzt werden kann, wenngleich Brod weiterhin eine Interessenkonvergenz mit den Tschechen postuliert. Mit diesem Konzept der Distanzliebe (der Begriff stammt aus Brods Roman Die Frau, die nicht enttäuscht) erfolgt aber zugleich eine als schmerzlich deklarierte Absage an Optionen ethnischer Assimilation:

Mein Verhältnis zum Deutschtum definierte ich als Kulturverbundenheit, denn aufs vertraulichste und entschiedenste war ich in deutscher Kultur erzogen worden, das bedeutete aber von nun an nicht mehr, daß ich mich dem deutschen Volk in eins zu verschmelzen hoffen durfte. Schmerzlicher Abschied, der mich durchtobte. [...] Es konnte Freundschaft mit dem deutschen Volk geben, Dankbarkeit für die von deutschen Genies geschaffenen geistigen Werte [...]. (Brod 1979: 52) $)^{12}$

Die für den Prager Kreis laut Johannes Urzidil charakteristischen deutschen, tschechischen jüdischen und österreichischen Einflussfaktoren, ${ }^{13}$ die immer auch eine Neutralisierung nationaler Polarisierung intendierten, bleiben zwar der Orientierungsrahmen für die kulturelle Vermittlung. In dem veränderten Kontext nach 1918 mit der Gründung eines tschechoslowakischen Nationalstaates, mit dem neue, auch kulturelle Grenzziehungen einhergingen, erhielt die Frage der Positionierung für die vor allem jüdischen Kulturmittler in Prag in einem sich partiell radikalisierenden nationalen Antagonismus eine erhöhte Virulenz. 1918 propagierte Max Brod sein Konzept der Kulturverwandtschaft durch »Sprache, Erziehung, Lektüre, Kultur«, welches eben keine »Blutsverwandtschaft « bedeute (Brod 1918: 1581f.) und das neben einer antihabsburgischen auch eine auf die Politik bezogene antideutsche Diktion verrät:

Judentum für sich entdeckte und in Auseinandersetzung mit Martin Buber und Hugo Bergmann seine "Philosophie der Mitte. Nicht der Mittelmäßigkeit" entwickelte (Vassogne 2009: 85).

12 | "[M]it diesem dialektischen, in sich Widerspruch und fruchtbare Spannung bergenden Terminus meinte ich, daß ich das Deutschtum, das deutsche Wesen liebte, doch mir zugleich einer gewissen Distanz von inm bewußt war, die mir beispielsweise verbot, fessellos scharfzüngige Kritik in der Art Tucholskys zu üben. Meine Kritik mußte von zurückhaltenderer, ernsthafterer Art sein. Diese meine diffizile, nach Kräften konsequent festgehaltene Einstellung hat mir sehr viel Feindschaft zugezogen, und zwar von deutscher wie von jüdischer Seite [...]. Ich blieb bei meiner Überzeugung, daß die Distanzen und Unterschiede der Völker, wenn sie an der Oberfläche unserer menschlichen Beziehung nur recht offen und klar ausgesprochen würden, eine desto innigere menschliche Gemeinschaft im Kern, in den allerwesentlichsten Zusammenhängen stiften würden. Diese neue Art dialektischen Zusammenhangs nannte ich eben Distanzliebe; ihr erhofftes Endresultat sollte ein alle Menschen umfassender Bund sein, der die Verschiedenheit der Völker nicht verschleiern, aber in verstehender Liebe überbrücken sollte und den ich den 'Nationalhumanismus nannte." (Brod 1979: 52f.)

13 | S. hierzu Urzidils Hinternationalitätskonzept`, mit dem er sich jenseits von "tschechischen, deutschen, jüdischen oder österreichisch-adligen « Zuschreibungen zu verorten suchte (Urzidil 1960: 12f.). 
Ich bejahe mein sprachliches Bedürfnis als deutschsprechender Jude. Ich werde nie anders als deutsch denken, schreiben, reden können [...]. Mein sprachliches Bedürfnis verlangt also, daß [...] in Prag gewisse deutsche Institutionen erhalten bleiben, Vereine mit deutscher Geschäftssprache, deutsche Vorlesungen und Vorträge, deutsches Theater, deutsche Presse, deutsche Schulen u.s.f. Dieses Bedürfnis hat sich dadurch, daß ich mich zum nationalen Judentum bekenne, nicht im mindesten geändert. Allerdings habe ich nur an der deutschen Sprachlichkeit dieser Institutionen Interesse, nicht an ihrer deutschen Politik [...]. (Ebd.: 1584)

Kulturelle Vermittlung verläuft somit als Form einer Grenzüberschreitung, die in transnationalen Zwischenräumen entsteht bzw. diese erst konstituiert, nicht aber als Dekonstruktion der nationalen Narrative und der an sie gekoppelten Nationalkulturen, z.B. über eine utraquistische oder supranationale Kultur. Max Brod ging es um Ausgleich zwischen den nationalen Antagonismen, um ein Wagnis der Mitte (Weltsch 1937), mit dem eine neutrale Stellung der jüdischen Bevölkerung unter Bewahrung eigenkultureller Traditionen zwischen Tschechen und Deutschen postuliert werden konnte.

\section{Strategien der Vermittlung}

Als Komponist, Musikkritiker, Bearbeiter von Opernlibretti, als Romanautor, Essayist, Journalist und Übersetzer, als - in heutiger Diktion - Netzwerker, verschrieb sich Brod aus ästhetischen, aber auch kulturpolitischen Gründen der kulturellen Vermittlung. Feldtheoretisch betrachtet setzte er sich für Werke und Künstler ein, die von einer normativ-zentralistischen Kunstkritik ausgegrenzt wurden. Ungeachtet von individuellen Vorlieben zeugen seine größten Leistungen auf dem Gebiet der Kunstvermittlung, also in Hinblick auf Leoš Janáček, Jaroslav Hašek und natürlich Franz Kafka, von fundierter ästhetischer Urteilskraft bzw. -kompetenz, aber eben auch von Einfluss auf das kulturelle Feld, auf dem es ihm - wenngleich gegen massive Widerstände ${ }^{14}$ - gelang, die Genannten nach und nach zu positionieren und durchzusetzen. In der Schaubühne veröffentlichte Brod seine Plädoyers für Gustav Mahler, Adolf Schreiber und Leoš Janáček, er wirkte ferner als Berichterstatter für den von Richard Batka und Richard Specht in Wien herausgegebenen Merker. Österreichische Zeitschrift für Musik und Theater (erschienen 1909-1922), später dann als Redakteur im offiziellen Prager Abendblatt sowie ab 1924 im Prager Tagblatt. ${ }^{15}$ Der frühe Text Tschechisches Opernglück in der

14 | Brod erwähnt, dass er anfänglich win tschechischen Fachkreisen wegen meines enthusiastischen Eintretens für den damals noch ganz verkannten Janáček" angegriffen wurde (Brod 1954: 13); vgl. hierzu auch Susskind 1985.

15 | Im Brief an Janáček vom 6. Februar 1924 kündigt Brod seinen Wechsel vom Staatsdienst zum Prager Tagblatt als Theater- und Musikreferent an ("An dieser Stelle werde ich viel mehr für Sie tun können als bisher." [Racek/Rektorys 1953: 137]) sowie die Absicht, "regelmäßig über tschechische Kunst und Kultur zu berichten, was gewiß 
Berliner Schaubühne, eine Art Paukenschlag, gilt als »der erste Schritt zu Janáčeks internationalem Erfolg« (Šrámková 2010: 77). Auf die gut dokumentierten Widerstände, die insbesondere Janáčeks Durchbruch blockierten, sei hier nicht ausführlicher eingegangen. Noch in den 1920er-Jahren, also nach großen Erfolgen, bezeichnete Josef Bartoš in Über Strömungen in der gegenwärtigen Musik (Prager Presse 1923: 71) Janáček als musikalischen Sonderling:

Sein Werk sollte man zuerst revidieren, bevor ein definitives Urteil gefällt wird. Janáček ist ein volkstümlicher Klugscheißer, der auch gerne auf Musiktheorie macht, er hat seine eigene Terminologie, an der er hängt, auch auf die Gefahr hin, daß man inn auslacht. Er hat das Volkslied studiert und von inm gelernt, er ist den Volksmelodien gefolgt und versucht nun, auf deren Grund den Charakter des Individuums zu erkennen. Er pflegt irgendeine Art Chiromantie oder Graphologie, und glaubt, daß er Musiktheorie studiere. (Zit. n. Knaus 1982: 71)

Welche Strategien der Kulturvermittlung lassen sich nun aber bei Max Brod identifizieren? Brod war zunächst ein regelmäßiger Rezensent, allein im Prager Tagblatt publizierte er 14 Artikel zu Janáček, hinzu kamen Artikel in der Prager Presse und im Prager Abendblatt, ferner im Berliner Börsencurier und in der NZZ (Briefe vom 26. April u. 30. Mai 1921). ${ }^{16}$ Er nahm zudem Kontakte zum Czechoslovak Press Bureau in Washington wegen Propagierung von Janáčeks Werk in den USA auf (vgl. Racek/Rektorys 1953: 77). Darüber hinaus übersetzte er die meisten seiner Libretti, wobei Brod den Text manchmal umfassend bearbeitete, um den Stoff besser in den deutschen Sprachraum vermitteln zu können, die Libretti also aus dem tschechischen Codesystem in das deutsche übertrug. Neben die Übersetzungen traten Kommentierung und Propagierung

ein großer Fortschritt im Sinne der Völkerverständigung ist." (Ebd.) Zu diesem Komplex s. auch Doležal 2004.

16 | Die Tabelle verzeichnet eine Übersicht der Artikel Max Brods in ausgewählten Periodika zwischen 1905 und 1939:

$\begin{array}{llr}\text { Die Gegenwart } & 1905-1911 & 67 \text { Artikel } \\ \text { Deutsche Arbeit } & 1906-1909 & 13 \text { Artikel } \\ \text { Die Schaubühne } & 1907-1920 & 36 \text { Artikel } \\ \text { Neue Rundschau } & 1908-1932 & 24 \text { Artikel } \\ \text { Die Aktion } & 1908-1917 & 48 \text { Artikel } \\ \text { Das literarische Echo } & 1910-1916 & 16 \text { Artikel } \\ \text { Weiße Blätter } & 1913-1916 & 12 \text { Artikel } \\ \text { Der Jude } & 1916-1928 & 14 \text { Artikel } \\ \text { Die Selbstwehr } & 1916-1938 & 118 \text { Artikel } \\ \text { Prager Abendblatt } & 1921-1923 & 355 \text { Artikel } \\ \text { Weltbühne/Neue Weltbühne } & 1921-1936 & 11 \text { Artikel } \\ \text { Prager Tagblatt } & 1924-1939 & 1872 \text { Artikel } \\ \text { Die literarische Welt } & 1926-1932 & 17 \text { Artikel }\end{array}$


des Werks auch in der Fachliteratur ${ }^{17}$ sowie biografische Arbeiten (Brod 1924). Schließlich pflegte Brod zahlreiche Kontakte zu Intendanten und Dirigenten, die er zur Durchsetzung von Janáčeks Werk einsetzte.

Aus Platzgründen kann der komplette Wirkungskreis der brodschen Mittlertätigkeit hier nicht dargestellt werden (vgl. hierzu Vassogne 2009 u. Šrámková 2010), erwähnt sei nur, dass Brod diese Aktivität auch zur Enttabuisierung wie im Falle des stigmatisierten Karel Sabina einsetzte. Wie bei Janáček, nur eben bei einem Toten, unternahm er den Versuch, einem bedeutenden Künstler zur verdienten Anerkennung zu verhelfen (Brod 1962). ${ }^{18}$

Rezeption und Wirkung kultureller Artefakte unterlagen, so Brod, den spezifischen Bedingungen einer kleinen Kultur und der ihr inhärenten Blockaden. Diese >Kleinheit< ist für Brod Grund für das Wirkungsdefizitit Janáčeks, ein Zeichen, dass auch ein

so kleines, sich selbst übersehendes Volk wie die Tschechen noch nicht die innere Kraft aufgebracht hat, in sich selbst Ordnung zu schaffen, das verderbliche Cliquenwesen zu beseitigen und die eigenen schöpferischen Potenzen an die richtigen Stellen zu setzen, obwohl dies [...] eine der wichtigsten nationalen und politischen Arbeiten wäre, sogar wichtiger als die welterschütternde Frage ein- oder doppelsprachiger Straßentafeln. (Brod 1966: 18f.)

Kulturvermittlung ist somit nicht nur ein Problem der sprachlichen Ebene, sondern auch eines der kulturpolitischen Hierarchisierung innerhalb der tschechischen Kultur. Das nationale Narrativ der kulturellen Emanzipation war an ein affirmatives Hochkulturverständnis gekoppelt, aus dem so genannte periphere Künstler mit lokal-volkstümlicher (Janáček) bzw. sozial-pejorativer (Hašek) Begründung genauso ausgeschlossen werden konnten wie politisch inkorrekte (der Konfident Karel Sabina). Zur sozialen wie kulturellen Isolierung Hašeks, der offenbar im tschechischen Verlagswesen nicht existent war, vermerkte Brod:

Ich wollte einmal eines seiner Bücher kaufen. Der Buchhändler nahm die Bestellung sichtlich ungnädig entgegen. Vorrätig sei das Buch nicht - und ehe es der Autor selbst bringen würde - kurz Garantie für einen bestimmten Lieferungstermin übernehme er nicht. [...] Ja, er ist gleichzeitig Verleger und Austräger seiner Werke. Aber er wohnt in Žižkov. Und zwischen Žižkov und dem Geschäft sind viele Stationen, [...] Sie verstehen doch: Wirtshäuser. Da trifft er dann vielleicht nicht her [...]. (Brod 1923: 6)

17 | Im Brief an Janáček vom 10. Juni 1926 verweist Brod auf seinen Janáček-Artikel für das englische Musiklexikon Cobett's Cyclopedia Survey of Chamber Musicl (Racek/ Rektorys 1953: 201).

18 | Karel Sabina, radikaler 1848er-Demokrat und Librettist von Smetanas Oper Prodaná nevěsta (Die verkaufte Braut), war ab 1859 Konfident der Prager Polizei. Nach seiner Enttarnung war er in der tschechischen Öffentlichkeit tabuisiert, das Libretto zur Verkauften Braut erschien in der Folge nur mit dem Kürzel "K.S.". 
Das heißt, Brod ging es nicht nur um Initiierung einer produktiven Rezeption außerhalb des tschechischen Kulturkontextes, sondern auch um Korrektur einer dogmatischen Rezeptionshaltung innerhalb, für die eine Kanonisierung in Korrelation zu nationaler Loyalität zu erfolgen habe. Bezogen auf Hašek ging es Brod um die Aufwertung eines volkstümlichen Charakters und damit der universellen Tragweite der Gestalt des Švejk, der auf eine Stufe mit Sancho Pansa und damit in einen weltliterarischen Kontext gestellt wird (vgl. Brod 1921: 6).

Hier lässt sich ein dezidiert kulturpolitischer Anspruch bei Max Brod erkennen, dem es um Durchsetzung von Werken im kulturellen Feld, um deren Kanonisierung, also ästhetische Aufwertung, Verleihung von Dauerhaftigkeit und Vorbildcharakter sowie um Monopolisierung, vor allem im Falle Franz Kafkas, und damit um Deutungshoheiten bzw. Instrumentalisierungen ging - nicht zuletzt im Hinblick auf das eigene Werk oder, wie im Falle Gustav Mahler, im Hinblick auf eine zionistische Integration von Kunst und Musik (vgl. Vassogne 2010). Es geht bei Max Brod also nicht um bloße Teilhabe wie bei Franz Werfel oder gar um ein Konzept >landestümlicher Symbiose wie bei Pavel Eisner (vgl. Wögerbauer 2011) oder auch Franz Spina (vgl. Höhne 2012), sondern eher um eine kulturpolitische Etablierung einer (fast) unbekannten Literatur und Musik, also deren intra- wie interkulturelle Valorisierung.

\section{Grenzen der Vermittlung}

Grenzen der Vermittlung lassen sich im Falle Max Brod auf drei Ebenen beobachten: auf a.) einer werkimmanenten, b.) einer aporetischen Ebene der Idealisierung von Kunst und c.) einer kulturpolitischen.

Die werkimmanente Ebene kann hier nur kurz angerissen werden. Im Hinblick auf Janáček zeigen sich bei den Bearbeitungen der Libretti drei zentrale Probleme (vgl. Doležal 2004). Diese betreffen zunächst den unterschiedlichen Wortakzent im Deutschen und Tschechischen. Sie sind ferner verursacht durch den Ehrgeiz Brods, eine Libretto-Prosa zu verfassen, die der musikalischen Dynamik auf das Genaueste folgte (vgl. Brod 1966: 35) und die von der Absicht geleitet war, im Sinne der Logik des Textes zu handeln. »Die Übersetzung muß nach meiner künstlerischen Überzeugung auch für sich ein Kunstwerk sein, also kein >Operndeutschく, sondern eine gesunde volkstümliche Sprache«, so Brod im Brief vom 13. Dezember 1916 (Racek/Rektorys 1953: 18). Daraus mussten Konflikte mit dem Komponisten entstehen, der auf einer wortgetreuen Übersetzung beharrte, was sich vor allem während der Arbeit an Věc Makropulos (Die Sache Makropulos) zeigte, wo der Komponist die allzu freie Wiedergabe, die nicht die »Härte des Originals« hatte, kritisierte (vgl. Šrámková 2010: 91). ${ }^{19}$ Und

19 | Vgl. hier den Brief von Janáček an Brod vom 2. Januar 1927 mit einer Auflistung von Unstimmigkeiten im Makropulos in der brodschen Übersetzung, u.a.: "Což nutno člověku tř ista let mezi lidmi žít?" ("Kann man es 300 Jahre unter Menschen aushalten?"); Brod: "Man muß den Namen in 300 Jahren öfter ändern."; "já už to nechci, zde 
schließlich spielen nationale Konnotationen eine Rolle. Brod kritisierte den zweiten Teil des Brouček wegen seiner lokalpatriotischen Färbung, undramatischen Gestaltung und Verherrlichung des Krieges, weshalb er dieses Libretto dann auch nicht übersetzte. Er schlug Änderungen vor (Brief v. 7. Juni 1920, zit. n. Racek/Rektorys 1953: 65), um das Libretto für das deutsche Publikum brauchbar zu machen:

Auch Religionskriege flößen mir Abscheu ein. - Ob der Krieg gegen Deutsche geführt wird oder nicht, ist Nebensache. Krieg ist Krieg. Den Krieg besingen ist so wie die Pest besingen. Nein, noch ärger! Für die Pest kann niemand, der Krieg aber ist ein Verbrechen beider Parteien. Beide haben Unrecht, so ist es immer! (Brief v. 17. Juli 1920, zit. n. Racek/Rektorys 1953: 68)

Brod (1966: 35), dem es um »Menschlichkeit, nicht Opernhaftigkeit! - Menschlichkeit, nicht Nationalitätenhaß!« ging, kollidiert hier offenkundig mit einer dezidiert nationalpatriotischen Einstellung Janáčeks.

Die zuvor erwähnte Forderung Brods weist auf eine Aporie der Vermittlung, die zwischen einem idealisierten Bild von Künstler und Kunst und den realen politisch-nationalen Einstellungen steht. Vergeblich bat Brod Janáček, öffentlich gegen den Antisemitismus aufzutreten.

Sie sagten mir einmal, daß Sie Verbindungen mit dem Venkov u. Večer haben. Könnten Sie nicht diesen Blättern nahelegen, etwas weniger antisemitisch zu schreiben? Es liegt gar nicht im Interesse der Tschechen, dem Antisemitismus zu frönen. Diese Dummheit sollten die Tschechen den politisch unreifen Deutschen überlassen! (Brief v. 30. Juli 1917, zit. n. Racek/Rektorys 1953: 34)

Immer wieder wies Brod in seinem Briefwechsel auf die antisemitischen Tendenzen in Prag hin, ohne dass eine Reaktion von Janáček zu finden ist. In einem Brief vom 30. April 1918 berichtet Brod: »Schrecklich ist aber der Antisemitismus in Deutschland. Er wird nur von dem Benehmen der tschechischen >Intelligenz< gegen die Juden übertroffen.« (Racek/Rektorys 1953: 47f.) Und am 20. Mai 1918 folgt ein Bericht vom Theaterfest auf dem Žofin in Prag und einer Begegnung mit Otokar Fischer:

Sofort sagt jemand, obwohl wir tschechisch miteinander sprachen: 'To jsou dva JeiteIesir. Eine drohende Menge sammelte sich und wir zogen es vor, wegzugehen. Auf der Gasse ruft man nicht 'Nieder mit den Deutschen`, sondern 'Nieder mit den Juden.' -

vemte si to" ("Ich will es nicht mehr, da nehmt es"); Brod: "Aber vom Tode verkostet ich heute schon." Im Brief vom 4. Januar 1927 verteidigt sich Brod, der sehr wohl auch inhaltliche Änderungen vornahm, im Sinne der Logik des Textes (Racek/Rektorys 1953). Zu den brodschen Übersetzungen s. zuletzt Horáčková 2007. 
Die tschechische Intelligenz hetzt das Volk gegen uns auf, - weil wir die schwächsten sind. (Racek/Rektorys 1953: 49) ${ }^{20}$

Auf der politischen Ebene offenbaren sich vor 1918 Aporien, die aus der unpolitischen Haltung Brods zu erklären sind, wobei er selbst auf der einen Seite seine nationalhumanistische Position nicht konsequent vertritt, da ethnische Kategorien als Identifikationsangebote nicht a priori verworfen werden. Brod fordert zum Beispiel, der Venkov möge »zwischen den deutschassimilierten Juden und den Jüdischnationalen, Zionisten unterscheiden lernen!« (Brief v. 30. Juli 1917, zit. n. Racek/Rektorys 1953: 35) Auf der anderen Seite verlegt er sich auf Illusionen: »Fliehen wir lieber in das Wunderreich der Kunst und Musik. Das Leben ist häßlich geworden.« (Brief v. 20. Mai 1918, zit. n. ebd.: 49) ${ }^{21}$ Diese partiell unpolitischen Züge, denen eine Position zugrunde liegt, nach der die Welt der Kunst einer anderen Logik zu folgen habe als die Welt der Politik, zeigen sich auch in der Folge, sei es in der naiven Betrachtung des Kommunismus (Grundlage ist hier eine Russlandreise 1935), sei es in dem natürlich zum Scheitern verurteilten Versuch, in eine intellektuelle Auseinandersetzung mit dem aufkommenden nationalsozialistischen Antisemitismus im Deutschen Reich zu treten. ${ }^{22}$

Vermittlungsprobleme auf werkimmanenter und kulturpolitischer Ebene, die beide die Authentizität des Kunstwerks tangieren, verraten auch Brods Ambitionen bei der Durchsetzung von Jaroslav Hašek. Hier sei nur kurz eine Dramatisierung des hašekschen Romans durch Brod und Hans Reimann genannt, bei deren Erstaufführung auf Piscators Berliner Bühne am Nollendorfplatz (23. Januar 1928) ein Konflikt zwischen dem Rechteinhaber Brod und dem Intendanten Piscator um die brodsche Fassung entbrannte. Piscator ging es um die Notwendigkeit eines politischen Theaters, während Brod und Reimann, die massiv in den Originaltext eingriffen, ein konventionelles Theaterstück schaffen wollten:

Da ein Theaterstück einen Schluß haben muß, erfanden wir eine durchlaufende Intrige, fügten einige Nebenfiguren ein, die bei Hašek nicht vorkommen, und ließen im Übrigen die wirksamsten Szenen des Romans in diesem neuen Gerüst abrollen (zit. n. Šrámková 2010: 247).

Die auf Prinzipien der Inklusion und Exklusion basierenden gesellschaftlichen Rahmenbedingungen und politischen Machtverhältnisse, die nicht nur auf einer sprachpolitischen Oberfläche verliefen, ${ }^{23}$ begrenzten die Handlungsspielräume

20 | Diesem Brief legte Brod den Artikel Židovské zprávy (Jüdische Nachrichten) aus dem České časopisectvo (1918/4: 5) bei.

21 | Zur Verklärung des Janáček-Bildes s. Belege bei Saremba 2001.

22 | Brod selbst konstatiert - ungeachtet einer gewissen Koketterie - eine gewisse Naivität bei seiner Flucht aus Prag in der Nacht vom 14. auf den 15. März 1939.

23 | Rückblickend relativiert Brod die sprachnationalen Konflikte vor 1938 als Folklore: "Doch es ist unmöglich, einem Nicht-Prager oder einem, der nicht jahrelang in Prag 
und setzten jeglicher Vermittlung Grenzen; eine die nationalen Konflikte neutralisierende, supranationale Kultur entstand, ungeachtet aller Austauschprozesse, nicht. Brods >Wagnis der Mitte<, mit der auch die neutrale Mittelstellung der Juden zwischen Tschechen und Deutschen postuliert wurde, konnte unter demokratisch-rechtsstaatlichen Bedingungen bedingt funktionieren, unter totalitären keinesfalls. Aus der Rückschau entwickelte Max Brod, in Erinnerung an die vielfältigen freundschaftlichen Beziehungen zwischen Tschechen und Deutschen in Prag sowie die verbreitete Sprachkompetenz, ${ }^{24}$ seinerseits ein Symbiosemodell, nach dem

in Prag kaum mehr von einer rein deutschen und einer rein tschechischen Nation die Rede ist, sondern nur noch von Pragern, Bewohnern dieser herrlichen und geheimnisvollen Stadt. Eine Verschmelzung ist eingetreten, das Blut hat sich vermischt, kulturelle und wirtschaftliche Beziehungen locken über die Grenzen. (Brod 1979a: 61)

Lässt sich auch auf der alltagskulturellen und -sprachlichen Ebene durchaus von einer multilingualen Sprachsituation mit unterschiedlichsten Interferenzerscheinungen und Mehrfachidentitäten sprechen, so muss vor Idealisierung gewarnt werden, denn die Krisenanfälligkeit solcher nicht-essentialistischer Identitätsangebote angesichts wachsender Dominanz und Akzeptanz des nationalen, zunehmend rassistisch-antisemitisch aufgeladenen Narrativs steht außer Frage. Ungeachtet vielfältiger Interaktionsorte von Cafés über Periodika bis hin zu Privathäusern (Salons), die als Kommunikationsräume des kulturellen Transfers fungierten, und ungeachtet intensiver wechselseitiger Rezeptionsund Wirkungsprozesse entstand eben keine supranationale Prager Literatur und Kultur. Prozesse der Entfremdung gegenüber der nun dominanten tschechischen Kultur scheinen gerade im Prager Kreis nach 1918 zu wachsen. ${ }^{25}$ Franz Kafka dokumentiert diese Erfahrung der Isolation und die damit verbundene Angst in einem Brief an Robert Klopstock Ende März 1923 weitaus realistischer, wenn er von seiner existentiellen Angst berichtet, die eben auch auf seine soziale Position bezogen wird: »Ein Jude und überdies deutsch und überdies krank und überdies unter verschärften persönlichen Umständen [...].« (Kafka 1975: $430)^{26}$

gelebt hat, die feinen und auch die unfeinen Varianten der Stellungnahme in der heißumstrittenen und historisch verwickelten Nationalitätenfrage klarzumachen, in der die Beschriftung jedes Ladenschildes und jeder Straßentafel ein Sprachenproblem, ein Politikum wurde." (Brod 1979a: 83)

24 | "Mit den Tschechen hielten wir gute Nachbarschaft und die tschechischen Dichter liebten wir; da gab es überhaupt nichts, was wie Grenze oder Absonderung abgesperrt hätte. Wir alle beherrschten die tschechische Sprache vollständig, die uns nicht weniger als die deutsche sagte." (Brod 1979a: 207)

25 | Hierzu die Umfrage "Warum haben Sie Prag verlassen?" (Krolop 2005: 89-102)

26 | Zur fehlgeschlagenen Assimilation schrieb Kafka im Juni 1921 an Max Brod: "Sie lebten zwischen drei Unmöglichkeiten, (die ich nur zufällig sprachliche Unmöglichkeiten nenne, es ist das Einfachste, sie so zu nennen, sie könnten aber auch ganz anders 


\section{FAZIT}

Was bleibt somit, wenn man ein Scheitern der kulturellen Vermittlung und den nur partiellen Eingang von Kulturmittlern wie Max Brod in das kollektive Gedächtnis aufgrund der Persistenz nationaler Deutungsmuster konstatieren muss? Langfristig und ungeachtet des bis heute kritisch diskutierten Engagements Brods, ob es sich um Bearbeitungen von Janáčeks Libretti oder die monopolistische Durchsetzung einer bestimmten Kafka-Interpretation handelt, bleibt das Verdienst, wie im Falle Janáček, einem Künstler, der »seine universelle Musik einer praktisch unbekannten Sprache geopfert « hatte (Kundera 1993: 180), zum Weltdurchbruch verholfen zu haben. Nimmt man die schopenhauersche Erklärung für Nachruhm als »die Summation der Urteile wirklich bedeutender Männer« (Brod 1966: 69), dann ist auch Max Brod nicht nur als Autor und Literat, sondern als Organisator, somit Vermittler von Literatur und Musik, der sich für Talente, von denen er überzeugt war, »tatkräftig und [häufig] erfolgreich einsetzte« (Schamschula 1987: 249), wiederzuentdecken.

\section{LiTERATUR}

Becher, Peter/Höhne, Steffen/Nekula, Marek (Hg.; 2012): Kafka und Prag. Literatur-, kultur-, sozial- und sprachhistorische Kontexte. Köln/Weimar/Wien.

Botstein, Leon (2003): The Cultural Politics of Language and Music: Max Brod and Leoš Janáček. In: Michael Beckerman (Hg.): Janáček and his World. Princeton, Oxford, S. 13-54.

Brod, Max (1918): Ein menschlich-politisches Bekenntnis. Juden, Deutsche, Tschechen. In: Die neue Rundschau 29, H. 2, S. 1580-1593.

Ders. (1921): Zwei Prager Volkstypen. Szenen von E.E. Kisch und J. Hašek im kleinen Theater Adria. In: Prager Abendblatt v. 7. November 1921, S. 6.

Ders. (1923): In memoriam Hašek. In: Prager Abendblatt v. 8. Januar 1923, S. 6.

Ders. (1924): Leoš Janáček. Život a dílo. Prag [dt. Ausgabe: Leoš Janáček. Leben und Werk. Wien 1925].

Ders. (1954): Janáčeks letzte Jahre. In: Heinrich Lindlar (Hg.): Musik der Zeit. Eine Schriftenreihe zur zeitgenössischen Musik. H. 8: Tschechische Komponisten. Bonn, S. 11-14.

Ders. (1962): Die Verkaufte Braut. Der Abenteuerliche Lebensroman des Textdichters Karel Sabina. München/Esslingen.

genannt werden): der Unmöglichkeit, nicht zu schreiben, der Unmöglichkeit, deutsch zu schreiben, der Unmöglichkeit, anders zu schreiben, fast könnte man eine vierte Unmöglichkeit hinzufügen, die Unmöglichkeit zu schreiben [...] also war es eine von alIen Seiten unmögliche Literatur, eine Zigeunerliteratur, die das deutsche Kind aus der Wiege gestohlen und in großer Eile irgendwie zugerichtet hatte, weil doch irgendjemand auf dem Seil tanzen muß." (Kafka 1975: 337f.) 
Ders. (1966): Prager Sternenhimmel. Musik- und Theatererlebnisse der zwanziger Jahre. Wien/Hamburg.

Ders. (1979): Streitbares Leben. Autobiographie 1884-1968. Frankfurt a.M.

Ders. (1979a): Der Prager Kreis. Mit einem Nachwort von Peter Demetz. Frankfurt a.M.

Doležal, Pavel (2004): Tomáš G. Masaryk, Max Brod und das Prager Tagblatt (19181938). Deutsch-tschechische Annäherung als publizistische Aufgabe. Frankfurt a.M.

Frankl, Michal (2011): „Prag ist nunmehr antisemitisch“. Tschechischer Antisemistismus am Ende des 19. Jahrhunderts. Übers. v. Michael Wögerbauer. Berlin.

Godé, Maurice/Le Rider, Jacques/Mayer, Françoise (Hg.; 1996): Allemands, Juifs et Tchèques a Prague. Deutsche, Juden und Tschechen in Prag 1890-1924. Montpellier.

Goffman, Erving (1993): Rahmen-Analyse. Ein Versuch über die Organisation von Alltagserfahrungen. 3. Aufl. Frankfurt a.M.

Höhne, Steffen (2000): Der Bohemismus-Diskurs zwischen 1800 und 1848/49. In: Brücken Germanistisches Jahrbuch Tschechien - Slowakei N.F. 8, S. 17-45.

Ders. (2012): Franz Spina. Ein Prager Slavist zwischen Universität und politischer Öffentlichkeit. In: Ders./Ludger Udolph (Hg.): Franz Spina (1868-1938). Ein Prager Slavist zwischen Universität und politischer Öffentlichkeit. Köln/Weimar/Wien, S. 9-37.

Ders./Udolph, Ludger (Hg.; 2010): Deutsche - Tschechen - Böhmen. Kulturelle Integration und Desintegration im 20. Jahrhundert. Köln/Weimar/Wien.

Horáčková, Květoslava (2007): Janáčkovy opery v překladech Maxe Broda. Brno.

Kafka, Franz (1975): Briefe 1902-1924. Hg. v. Max Brod. Frankfurt a.M.

Ders. (2013): Briefe 1918-1920. Hg. v. Hans-Gerd Koch. Frankfurt a.M. [= Franz Kafka: Schriften, Tagebücher, Briefe. Kritische Ausgabe].

Knaus, Jakob (1982): Leoš Janáček. Materialien. Aufsätze zu Leben und Werk. Zürich.

Koeltzsch, Ines (2011): Zwischen den Kulturen. Zur Einführung. In: Dies./Michaela Kuklová/Michael Wögerbauer (Hg.): Übersetzer zwischen den Kulturen. Der Prager Publizist Paul/Pavel Eisner. Köln/Weimar/Wien, S. 9-15.

Krolop, Kurt (2005): Studien zur Prager deutschen Literatur. Hg. v. Klaas-Hinrich Ehlers, Steffen Höhne u. Marek Nekula. Wien.

Kundera, Milan (1993): Verratene Vermächtnisse. München.

Němec, Mirek (2010): Erziehung zum Staatsbürger? Deutsche Sekundarschulen in der Tschechoslowakei 1918-1938. Essen.

Racek, Jan/Artuš Rektorys (Hg.) (1953): Korrespondence Leoše Janáčka s Maxem Brodem. Prag.

Saremba, Meinhard (2001): Leoš Janáček. Zeit, Leben, Werk, Wirkung. Kassel u.a.

Schamschula, Walter (1987): Max Brod und die tschechische Literatur. In: Margarita Pazi (Hg.): Max Brod 1884-1984. Untersuchungen zu Max Brods literarischen und philosophischen Schriften. New York u.a., S. 233-249.

Schmitz, Walter/Udolph, Ludger (Hg.; 2001): Tripolis Praga. Die Prager Moderne um 1900. Katalogbuch. Dresden.

Šrámková, Barbora (2010): Max Brod und die tschechische Kultur. Wuppertal.

Susskind, Charles (1985): Janáček and Brod. New Haven/London.

Tramer, Hans (1964): Prague - City of Three People. In: Leo Baeck Institute Yearbook 9, S. 305-339. 
Urzidil, Johannes (1960): Prager Triptychon. Zürich.

Vassogne, Gaëlle (2009): Max Brod in Prag: Identität und Vermittlung. Tübingen.

Weltsch, Felix (1937): Das Wagnis der Mitte. Ein Beitrag zur Ethik und Politik der Zeit. Mährisch-0strau.

Werfel, Franz (1980): Zwischen Oben und Unten. Prosa, Tagebücher, Aphorismen, Literarische Nachträge. Aus dem Nachlaß hg. v. Adolf D. Klaarmann. München/Wien.

Werner, Michael/Zimmermann, Bénédicte (2002): Vergleich, Transfer, Verflechtung.

Der Ansatz der Histoire croisée und die Herausforderung des Transnationalen. In: Geschichte und Gesellschaft 28, H. 4, S. 607-636.

Wögerbauer, Michael (2011): Mediale Strategien der Vermittlung im Wandel. Eine quantitative Analyse von Paul Eisners Beiträgen in tschechoslowakischen Periodika (1918-1938). In: Ines Koeltzsch/Michaela Kuklová/Ders. (Hg.): Übersetzer zwischen den Kulturen. Der Prager Publizist Paul/Pavel Eisner. Köln/Weimar/Wien, S. 219-232. 